\title{
Evaluation of Weather Research and Forecasting (WRF) Microphysics single moment class- 3 and class- 6 in Precipitation Forecast
}

\author{
Syeda Maria Zaidi1 ${ }^{1,}$, Jacqueline Isabella Anak Gisen ${ }^{1,2}$ \\ ${ }^{1}$ Faculty of Civil Engineering \& Earth Resources, University Malaysia Pahang, Gambang, Malaysia \\ ${ }^{2}$ Centre for Earth Resources Research \& Management (CERRM), University Malaysia Pahang, Gambang, Malaysia.
}

\begin{abstract}
In this study, the performance of two different Microphysics Scheme options in Weather Research and Forecasting (WRF) model were evaluated for the estimating the precipitation forecast. The schemes WRF single moment class-3 (WSM-3) and single moment class-6 (WSM-6) were employed to produce the minimum, medium and maximum precipitation for the selected events over the Kuantan River Basin (KRB). The obtained simulated results were compared with the observed data from eight different rainfall gauging stations. The results comparison indicate that WRF model provides better forecasting at some rainfall stations for minimum and medium rainfall events but did not produce good result during maximum rainfall overall. The WSM-6 scheme is found to produce better result compared to WSM-3. The study also found that to acquire accurate precipitation results, it is also required to test some other physics scheme parameterization to enhance the model performance.
\end{abstract}

\section{Introduction}

The new generation Weather Research and Forecasting (WRF) model is the collaborative product of National Central of Atmospheric Research (NCAR) the mesoscale division, the national environmental prediction and forecast system lab (FSL) of Nation Oceanic and Atmospheric Administration (NOAA), the Naval Research Laboratory (NRL), the Department of Defence's Airforce Weather Agency (AFWA) and the Centre for Analysis and Prediction of Storms (CAPS) from the University of Oklahoma and the contribution of number of research scientists along with Federal Aviation Administration (FAA). The model is designed for both operational forecasting and weather research [1].

The WRF model is suitable for broad spectrum forecasting applications ranging from meters to thousands of kilometre. WRF contains many configured physics schemes to be easily implemented by the model user which include the advantages such as economical computation, existing default parameters, and relatively large spatial resolution grid. The offered numerous physics schemes in WRF are varies from simple to more sophisticate and computationally costly [2]. The physical schemes in WRF play a crucial role in WRF modelling. The parameterization of the schemes typically depends on the objective of the experiment design and the features of study area. The definition of parameterization refers to the process of selecting required parameters for a specific model. Besides that, parameterization in WRF also describes the phenomena occurs at sub gird scale. A significant amount of research has been conducted to evaluate the influence of physics parameterization and sensitivity of WRF in meteorological scenarios [3-8]. The parameterization of schemes is an important aspect related to the model forecasting accuracy. The blending of schemes depends on the domain of the research selection.

Since the last decades, a broad spectrum of physical parameterization schemes have been executed in weather models. Every scheme was developed based on different criteria which serve as a key source to identify the model's constraint and inaccuracies [9]. However, to define the optimum parameterization of every physics schemes is a highly difficult task as the number of these schemes have been increasing. Hence, this paper aims to to evaluate the performance two available Microphysics scheme options; single moment class -3 (WSM-3) and single moment class-6 (WSM-6) to determine the physical characteristic of precipitation with in the clouds.

\subsection{Microphysics Scheme WSM class-3 and WSM class-6}

Microphysics scheme plays a vital role in clouds, climate, and climatic models. It is used to regulate the process of water vapour and the formation of raindrops, hail and snow inside or outside the clouds [10]. It is also used to determine the type, size, and distribution of precipitation. The scheme is essentially required to attain accurate rainfall simulation output. Currently, the WRF system is supporting 13 microphysics options which range from simple to more complex physical options depending upon the criteria of a model designed and area

* Corresponding author: mariazaidi26@gmail.com 
topography. In order to determine the physical characteristics of precipitation WRF Microphysics parameterization can be applied by simple scheme single moment class or advance scheme double moment [11].

The single moment scheme uses as a single prediction equation for each moment of precipitation to determine the particle size distribution. Whereas, the double moment is used to regulate the number and concentration of double moment of precipitation with an additional process; to determine the influence of aerosol on clouds [12]. The single moment WSM-3 is a basic simple option which has clouds formation by considering three states of water namely cloud water, ice water and rainwater [13].This scheme is computationally efficient and simple suitable for meso-scale grid. On the other hand, The WSM-6 scheme is suitable for high resolution gird. The scheme class- 6 has ice, snow, and rain with the extended association of graupel processes. The scheme is also suitable to use for cloud resolving grids [14].

\section{Material \&Method}

\subsection{Description of Study Area}

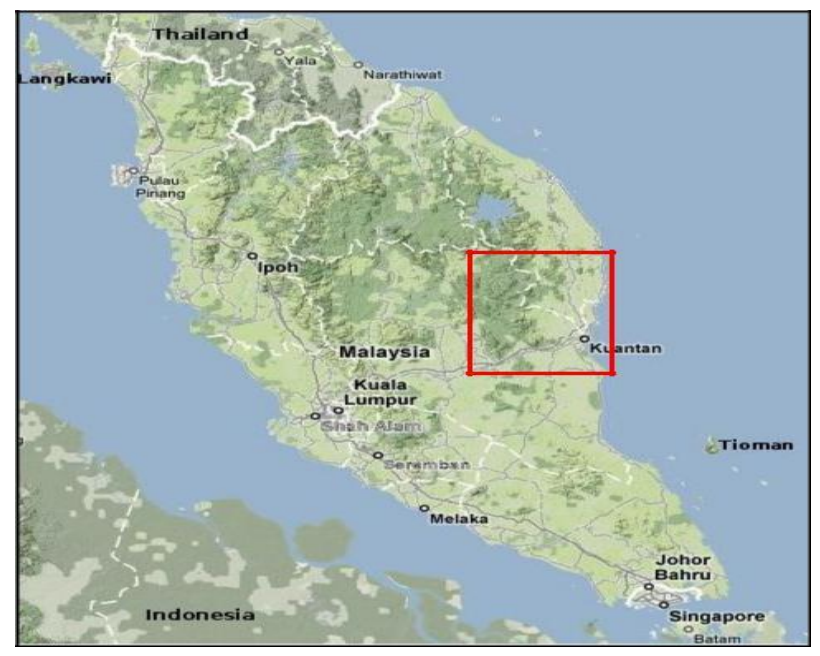

Fig. 1. Location of Kuantan River Basin

Kuantan River Basin (KRB) is the one of most important river basin located at the north east end of Pahang state in the peninsular Malaysia. KRB with the catchment area of $1630 \mathrm{~km} 2$ starts from $\mathrm{Sg}$. Lembing passing through Kuantan city and ends at the South China Sea. KRB has tropical climatic condition. The area has experienced massive precipitation during the period of north east monsoon season from October to March which caused spilling over of river and produce floods. Additionally the Kuantan River is the main source of water supply in that region.

\subsection{Data and Design of Experiment}

In this study, the observed daily precipitation data records were obtained from the Department of Drainage and Irrigation (DID) Kuantan. The dataset was used to validate the accuracy of WRF model precipitation forecast at eight different rainfall gauging stations. The performance of the selected two microphysics options in the model was evaluated by comparing the simulated precipitation with gauged rainfall data for three different events. The selection of events and categorization of precipitation was based on minimum, medium and maximum recorded rainfall. The study has chosen 10th Jan 2012 as the minimum received rainfall, whereas, the rainfall events on 11th and 12th Jan 2012 set as medium and maximum precipitation respectively. WRF model version 3.01 was setup the with horizontal outer domain of grid resolution at $36 \mathrm{~km}(\mathrm{~d} 01)$, inner domain (d02) at $12 \mathrm{~km}$ resolution and domain $(\mathrm{d} 03)$ at $4 \mathrm{~km}$ resolution grid, as shown in Fig.2. The Peninsular Malaysia is covered at $12 \mathrm{~km}$ gird resolution. The boundary condition for all the simulation used in this study was $10 \mathrm{x} 10$ re-analysis data from the National Centre for Environmental Prediction (NCEP) Global Final Analysis (FNL). In total 6 simulations were conducted for both microphysics schemes (WSM-3 and WSM-6) with combination of other selected physics options for the study as shows in the given table (1).

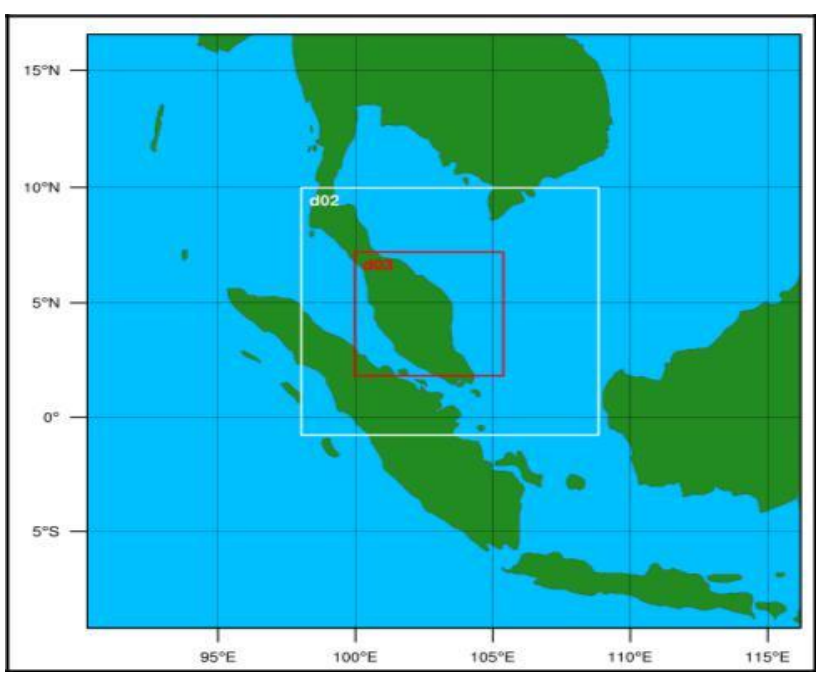

Fig. 2. WRF Model Grid Domains

Table 1. Selected WRF Model Physics

\begin{tabular}{|l|l|}
\hline \multicolumn{1}{|c|}{ Physics Scheme } & \multicolumn{1}{|c|}{ WRF Option } \\
\hline Cumulus & Betts-Miller_Jinjic (BMJ) \\
\hline Longwave Radiation & $\begin{array}{l}\text { Rapid radiative transfer } \\
\text { model (RRTM) }\end{array}$ \\
\hline Shortwave Radiation & $\begin{array}{l}\text { Dhudiha Scheme MM5 short } \\
\text { wave }\end{array}$ \\
\hline Surface Layer & $\begin{array}{l}\text { Monin-Obukhov similarity } \\
\text { theory }\end{array}$ \\
\hline Planetary boundary Layer & PBL scheme (MRF) \\
\hline
\end{tabular}




\section{Results}

The impact of two-available microphysics schemes in WRF model were tested for three different rainfall scenarios. Fig.3. shows the simulated precipitation which was conducted for minimum rainfall event on 10th Jan 2012 at different gauging stations. The result indicates that WRF model estimate uncertain forecasting values at different gauge locations. In comparison to the WSM-6, the scheme WSM-3 overestimated the rainfall at station Paya Besar, Kg. Sg Soi and Pulau Manis and lowest rainfall produced at Rumah Pam. However, both the scheme produced significantly rain accuracy at Ladang Kuala Raman compared to the observed rain.

In the in case of medium rainfall event on 11th Jan 2012 as shown in Fig.4. The simulated rainfall from WSM-3 produced slightly higher rain rate at stations Paya Besar and JPS Negeri Pahang. However, for the other stations both the WSM-3 and WSM-6 schemes have underestimated rainfall compared to the observed.

Fig 5. Shows the maximum predicted precipitation values from the model, simulated for the date 12th Jan 2012. The performance of the WRF model seems to be not satisfactory in producing heavy precipitation forecast. Although the schemes WSM-3 and WSM-6 shows the same pattern of forecasting but both the schemes did not produce compatible precipitation result to the observed for all the rainfall stations. Despite the fact, the microphysics scheme WSM-6 was found to produce the slightly substantial level of performance in all simulations output compared to WSM-3.

\section{Discussion}

Considering all three rainfall scenarios, It found the microphysics scheme single moment class 6 (WSM-6) parameterized with other given physics option in WRF is able to produce better result in simulating minimum and medium precipitation events. Nevertheless, this scheme parameterization cannot be employed for all rainfall station as shown in the results. This may cause by the variation in topographic characteristics and atmospheric properties at certain stations. Therefore, it is necessary to test other model physics options to find the best suitable combination of parameterized scheme for the area. With regards to the model underestimation in simulating heavy rainfall, there has been numerous influenced factors which play key role to the WRF model accuracy. For instance, the selection of domain size, down scaling of lateral boundary conditions as well as the parameterization of appropriate physics scheme options, topographic features and climatic condition of the region.

This study tested the effects of two microphysics options WSM-3 and WSM-6 over the WRF model performance. For that reason, model simulations results found to be uncertain and inappropriate to produce precipitation forecast. Therefore, to obtain better WRF performance, it is also important to consider other influential model factors as discussed.

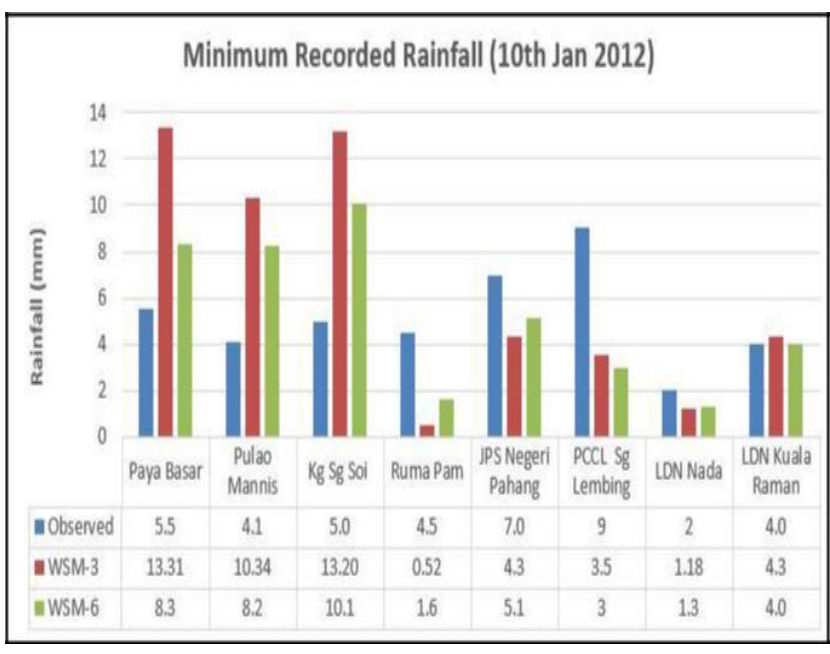

Fig 3. Minimum Simulated and Recorded Rainfall on $10^{\text {th }}$ Jan 2012

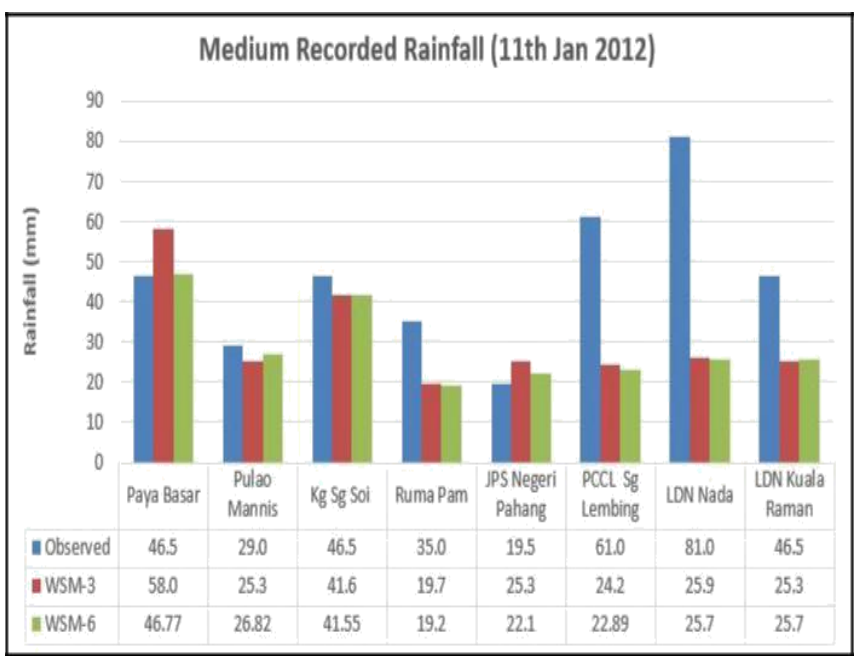

Fig. 4. Medium Simulated and Recorded Rainfall on 11th Jan 2012

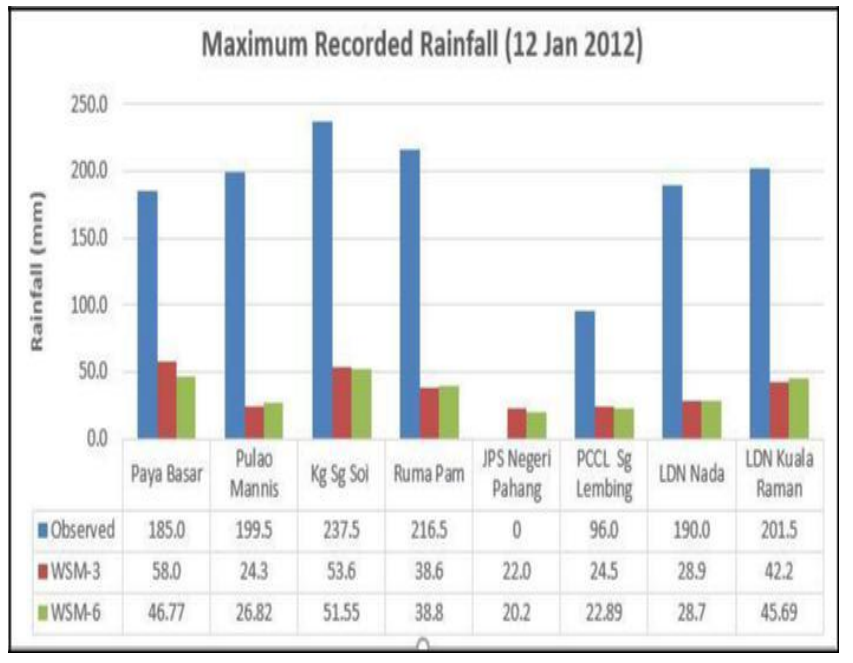

Fig. 5. Maximum Simulated and Recorded Rainfall on 12th Jan 2012 


\section{Conclusion}

Microphysics scheme plays a vital role in atmospheric science. It has the significant role to deal with process of vapour, clouds and precipitation. In other words, microphysics scheme is one the responsible factor to produce rainfall. WRF model has available 13 microphysics options which are used to determine the formation, distribution and concentration of vapours, rain/snow, ice and clouds. This study was aimed to determine the precipitation forecast pattern of WRF model at Kuantan River employed by two simple options of microphysics single class (WSM-3 and WSM-6). The model was designed to simulate three different events to produced minimum, medium and maximum rate of rainfall. The event 10th Jan2012 was selected for minimum precipitation whereas, 11 th Jan 2012 and 12th Jan 2012 events were selected for medium and maximum precipitation respectively. The result was validated from the observed rainfall at eight rainfall stations. From the study outcomes, it can be conclude that although microphysics Scheme WSM-6 showed better performance compared to the WSM-3 but it still produced uncertainties in results at different rainfall stations. However for the maximum precipitation forecast, both the schemes provided underestimate precipitation forecast. Considering the outcome of the study, it is cautious to state the exact performance of both schemes in forecasting heavy precipitation. This is because the study was focused on very specific objective. Therefore, more experiments are required to conduct considering other model factors including different physics option parameterizations, appropriate boundary condition and gird resolution to enhance the WRF model accuracy.

The authors are grateful to the University Malaysia Pahang (UMP) for the financial support of this research through the $\begin{array}{lll}\text { grant numbers } & \text { RDU150127 and }\end{array}$ FRGS/1/2015/WAB08/UMP/02/1.

\section{References}

1. Michalakes, J., et al. The weather research and forecast model: software architecture and performance. in Proceedings of the Eleventh ECMWF Workshop on the Use of High Performance Computing in Meteorology. 2005. World Scientific: Singapore.

2. Xue, Y., et al., A review on regional dynamical downscaling in intraseasonal to seasonal simulation/prediction and major factors that affect downscaling ability. Atmospheric research, 2014. 147: p. 68-85.

3. Bukovsky, M.S. and D.J. Karoly, Precipitation simulations using WRF as a nested regional climate model. Journal of applied Meteorology and Climatology, 2009. 48(10): p. 2152-2159.
4. De Silva, G., et al. Application of WRF with different cumulus parameterization schemes for precipitation forecasting in a tropical river basin. in Proceedings of the 13th Asian Congress of fluid Mechanics. 2010.

5. Maussion, F., et al., WRF simulation of a precipitation event over the Tibetan Plateau, Chinaan assessment using remote sensing and ground observations. Hydrology and Earth System Sciences, 2011. 15(6): p. 1795.

6. Sohrabinia, M., W. Rack, and P. Zawar-Reza, Analysis of MODIS LST compared with WRF model and in situ data over the Waimakariri River basin, Canterbury, New Zealand. Remote Sensing, 2012. 4(11): p. 3501-3527.

7. Im, E.-S., S.-R. In, and S.-O. Han, Numerical simulation of the heavy rainfall caused by a convection band over Korea: a case study on the comparison of WRF and CReSS. Natural Hazards, 2013. 69(3): p. 1681-1695.

8. Mooney, P., F. Mulligan, and R. Fealy, Evaluation of the sensitivity of the weather research and forecasting model to parameterization schemes for regional climates of Europe over the period 1990-95. Journal of Climate, 2013. 26(3): p. 1002-1017.

9. Awan, N.K., H. Truhetz, and A. Gobiet, Parameterization-induced error characteristics of MM5 and WRF operated in climate mode over the Alpine region: an ensemble-based analysis. Journal of Climate, 2011. 24(12): p. 3107-3123.

10. Hong, S.-Y., J. Dudhia, and S.-H. Chen, A revised approach to ice microphysical processes for the bulk parameterization of clouds and precipitation. Monthly Weather Review, 2004. 132(1): p. 103-120.

11. Dudhia, J., WRF modeling system overview. www2.mmm.ucar.edu/wrf/users/tutorial/201201/W RF_Overview_Dudhia. ppt. pdf, 2014.

12. Skamarock, W., Coauthors, 2008: A description of the advanced research WRF version 3. NCAR Tech. Note. NCAR/TN-475+ STR.

13. Dudhia, J., Numerical study of convection observed during the winter monsoon experiment using a mesoscale two-dimensional model. Journal of the Atmospheric Sciences, 1989. 46(20): p. 3077-3107.

14. Hong, S.-Y. and J.-O.J. Lim, The WRF singlemoment 6-class microphysics scheme (WSM6). J. Korean Meteor. Soc, 2006. 42(2): p. 129-151. 